\title{
Three Patients With Disseminated Mycobacterial Infections Due to Severe Defects in Interferon Gamma Receptor Signaling: A Challenging Diagnosis
}

\section{Zijun Zhou}

University Medical Center Rotterdam

Iris H.I.M. Hollink

University Medical Center Rotterdam

Arjan Bouman

University Medical Center Rotterdam

Mirthe S. Lourens

University Medical Center Rotterdam

Rik A. Brooimans

University Medical Center Rotterdam

Tjakko J. van Ham

University Medical Center Rotterdam

Pieter L.A. Fraaij

University Medical Center Rotterdam-Sophia Children's Hospital

Annemarie M.C. van Rossum

University Medical Center Rotterdam-Sophia Children's Hospital

Eline A.M. Zijtregtop

University Medical Center Rotterdam-Sophia Children's Hospital

Willem A. Dik

University Medical Center Rotterdam

Virgil A.S.H. Dalm

University Medical Center Rotterdam

P. Martin van Hagen

University Medical Center Rotterdam

Hanna IJspeert

Erasmus MC https://orcid.org/0000-0002-7061-5321

Clementien L. Vermont ( $\nabla$ c.vermont@erasmusmc.nl )

University Medical Center Rotterdam 


\section{Research Article}

Keywords: mendelian susceptibility to mycobacterial disease, IFN-gamma receptor, STAT1, mycobacterial infection, SOCS1

Posted Date: July 9th, 2021

DOI: https://doi.org/10.21203/rs.3.rs-682108/v1

License: (c) (i) This work is licensed under a Creative Commons Attribution 4.0 International License.

Read Full License 


\title{
Three patients with disseminated mycobacterial infections due to severe defects in interferon gamma receptor signaling: a challenging diagnosis
}

\author{
Zijun Zhou, ${ }^{1,2,6}$ Iris H.I.M. Hollink, ${ }^{3,6}$ Arjan Bouman, ${ }^{3,6}$ Mirthe S. Lourens, ${ }^{1,6}$ Rik A. Brooimans,,${ }^{1,6}$ \\ Tjakko J. van Ham, ${ }^{3}$ Pieter L.A.Fraaij, ${ }^{4,6}$ Annemarie M.C. van Rossum, ${ }^{4,6}$ Eline A.M. Zijtregtop, ${ }^{5}$ \\ Willem A. Dik, ${ }^{1,6}$ Virgil A.S.H. Dalm,,${ }^{1,2,6}$ P. Martin van Hagen,,${ }^{1,2,6}$ Hanna IJspeert, ${ }^{1,6 \#}$ Clementien \\ L. Vermont ${ }^{4,6 \#}$ \\ ${ }^{1}$ Erasmus MC, University Medical Center Rotterdam, Laboratory Medical Immunology, department of Immunology, the \\ Netherlands \\ ${ }^{2}$ Erasmus MC, University Medical Center Rotterdam, department of Internal Medicine, Division of Clinical Immunology, the \\ Netherlands \\ ${ }^{3}$ Erasmus MC, University Medical Center Rotterdam, department of Clinical Genetics, the Netherlands \\ ${ }^{4}$ Erasmus MC, University Medical Center Rotterdam-Sophia Children's Hospital, department of Pediatrics, Division of Pediatric \\ Infectious Disease and Immunology, the Netherlands \\ ${ }^{5}$ Erasmus MC, University Medical Center Rotterdam-Sophia Children's Hospital, department of Pediatrics, Division of Pediatric \\ Hemato-oncology, the Netherlands \\ ${ }^{6}$ Erasmus MC, University Medical Center Rotterdam, Academic Center for Rare Immunological Diseases (RIDC), the \\ Netherlands
}

\# these authors contributed equally

Corresponding author:

Dr. Clementien L.Vermont

Erasmus MC University Medical Center-Sophia Children's Hospital

PO Box 2060

$3000 \mathrm{CB}$

Rotterdam

The Netherlands

c.vermont@erasmusmc.nl 


\begin{abstract}
IFN-gamma receptor (IFNGR) signaling via STAT1 is crucial in the defense against intracellular pathogens. Defects in this pathway enhance the susceptibility to infection by otherwise weak pathogenic mycobacteria, resulting in a primary immunodeficiency called mendelian susceptibility to mycobacterial disease (MSMD).
\end{abstract}

Here we describe three patients with MSMD caused by variants in the IFNGRI or STAT1 genes. All three patients presented with disseminated non-tuberculous mycobacterial infections caused by $M$. avium, M. persicum or M. bovis BCG respectively. Whole-exome sequencing (WES) was used as the first line diagnostic approach, however in all patients additional analysis was crucial to make the definite diagnosis. In Patient 1, only one heterozygous autosomal recessive variant p.(Val63Gly) in the IFNGR1 gene was identified. Patient 2 was compound heterozygous for the pathogenic null p.(Val68Lysfs*6) variant and the hypomorphic p.(Ile37Thr) variant in IFNGRI. In Patient 3 a novel variant in the STAT1 gene c.1379A $>$ T, p.(Asn460Ile) was identified. Additional genetic analysis identified a second novel complex Alu-insertion in the IFNGR1 gene in Patient 1. Functional analysis showed that Patients 1 and 2 had reduced expression of IFNGR1. All patients had reduced phosphorylation of STAT1 and absent induction of SOCS1 mRNA after IFN- $\gamma$ stimulation. While STAT1 phosphorylation was normal after IFN- $\alpha$ stimulation in Patient 1 and 2, it was mildly reduced in Patient 3.

We conclude that functional assays are crucial to assess the extent of IFNGR signaling defects when new combinations of bi-allelic or non-conclusive genetic variants are found, which is important in the determination of clinical treatment.

Key words: mendelian susceptibility to mycobacterial disease, IFN-gamma receptor, STAT1, mycobacterial infection, SOCS1 


\section{Introduction}

Mendelian susceptibility to mycobacterial disease (MSMD) is a rare inherited disorder characterized by infection with weakly virulent mycobacteria including Calmette-Guérin bacillus (BCG) in otherwise healthy individuals $(1,2)$. Mycobacterial disease usually begins in childhood and less frequently in adolescence and adulthood. Patients are not restricted to susceptibility to mycobacterial species only, as they are also vulnerable to salmonellosis, candidiasis and tuberculosis (2).

In 1996, the first variant associated with MSMD was reported. It was described as bi-allelic null variants in interferon- $\gamma$ receptor 1 (IFNGRI) $(3,4)$. Since then, MSMD-causing variants have been reported in various autosomal genes such as IFNGR1, IFNGR2, IFNG, IL12B, IL12RB1, IL12RB2, STAT1, IRF8, ISG15, SPPL2A, TYK2, and two X-linked genes: NEMO and CYBB (or gp91phox) (5-14). These genes all encode proteins involved in the IL12/23-IFN- $\gamma$ signaling pathway: they are either involved in controlling the production of IFN- $\gamma$ (IL12B, IL12RB1, IL12BR2, IRF8, ISG15, NEMO) or the response to IFN- $\gamma$ (IFNGR1, IFNGR2, STAT1, IRF8, TYK2, CYBB).

This pathway is pivotal to the innate immune defense against mycobacteria, bridging between myeloid cells and lymphoid cells (15). Upon mycobacterial infection, mononuclear phagocytes produce interleukin (IL) 12, which activates T cells and natural killer cells via the IL-12 receptor, an IL12RB1 and IL12RB2 heterodimer. Upon ligand binding the IL-12 receptor signals through TYK2 and JAK2, resulting in STAT4 phosphorylation, followed by STAT4 homo-dimerization and nuclear translocation and finally IFN- $\gamma$ production. In response, IFN- $\gamma$ binds to its receptor, IFNGR, a heterodimer of IFNGR1 and IFNGR2 chains. This ligand-receptor binding leads to downstream phosphorylation of JAK2, JAK1, and STAT1. Homodimer phosphorylated STAT1 (pSTAT1) will translocate into the nucleus and binds to the IFN- $\gamma$ activation sequence (GAS) elements, enhancing transcription of IFN- $\gamma$-responsive genes. These series of events will eventually enable macrophage activation, differentiation, and further upregulation of proinflammatory cytokine production, including IL-2 and TNF- $\alpha$ (16). Signaling via IFN- $\gamma$ is tightly regulated by the SOCS (Suppressors of Cytokine Signaling) 1 protein, which acts as a negativefeedback inhibitor (17). 
IFNGR1 and IL12RB1 deficiency are the most common causes of MSMD, accounting for almost $80 \%$ of all genetically diagnosed cases (14). The pathogenic variants in IFNGR1 can be either autosomal recessive (OMIM \#209950) or dominant (OMIM \#615978) (18), and can result in complete or partial functional defects $(3,4,19,20)$. Lack of surface expression leads to a complete loss of function, while expression of mutated IFNGR1 can lead to either partial loss of function or complete loss of function. Complete loss of function in IFNGR signaling results in severe mycobacterial infections in early childhood and poor survival. However, variants with partial loss of function may result in a milder clinical phenotype that manifests later in childhood.

In this manuscript, we describe 3 patients with presentation of MSMD with novel (combination of) variants in genes involved in the IFN- $\gamma$ STAT1 signaling pathway. These cases illustrate the clinical and diagnostic challenges that can be faced, and show that additional genetic and functional immunological assays help to explain the clinical phenotype and can be helpful in the diagnostics of suspected IFNGR signaling defects in patients. 


\section{Methods}

\section{Ethical approval}

Following approval from the Erasmus MC Medical Ethics Committee (MEC-2013-026, MEC2016-606, or MEC-2016-202), written informed written consent was obtained from all parents and healthy controls before blood donation in accordance with the Declaration of Helsinki.

\section{Genetic analysis}

Genomic DNA of all three patients was extracted from peripheral blood samples using standard procedures. A diagnostic WES-based PID gene panel analysis was performed, which included around 400 PID-associated genes based on the IUIS classifications 2017 or 2019 (21, 22). DNA was enriched for the exome using the Agilent Sureselect Human All Exon V7 (Patient 1 and 2) or Agilent Sureselect Clinical Research Exome V2 (Patient 3) Capture Enrichment kits (Agilent Technologies) and paired-end sequenced on the Illumina Hiseq platform (GenomeScan, Leiden, the Netherlands) aiming at an average coverage of the exome of $\sim 50 \mathrm{X}$. Data were demultiplexed by Illumina software bclfastq, sequence reads were mapped to the genome with the BWA algorithm (http://bio-bwa.sourceforge.net/) and variant calling was performed by the Genome Analysis Toolkit (http://www.broadinstitute.org/gatk/). Detected variants in the PID-associated genes were filtered and annotated with the Cartagenia software package and classified with Alamut Visual. The detected IFNGR1 and STAT1 variants were confirmed by Sanger sequencing.

Diagnostic chromosomal investigation in P1 was performed using high-resolution SNP-array (Illumina Infinium ${ }^{\mathrm{TM}}$ Global Screening Array-24 v3.0 BeadChip).

Long range PCR was performed on DNA from patient 1 to amplify the genomic region surrounding exon 3 using the forward primer 5'- CCTGGTGAATTCTACTTTTCTTCAA-3' and the reverse primer 5' - AGTGTTTCTTAAGCATTGTGATAATTT-3' using Phusion high fidelity DNA polymerase (Thermo Fisher, Waltham, US). The longer PCR product in Patient 1, which was not observed in the healthy controls, was extracted from gel and purified using the gel extraction kit (Qiagen, Hilden, Germany). The purified band was sequenced and analysed using CLC main workbench 7 (Qiagen). The sequence of the insertion was uploaded in RepeatMasker (https://www.repeatmasker.org/cgi-bin/WEBRepeatMasker) (23). 


\section{IFN-gamma and IFN-alpha stimulation}

Peripheral blood mononuclear cells (PBMCs) were isolated from fresh blood from 3 patients and healthy controls via density gradient centrifugation (Ficoll-Hypaque, GE Healthcare life sciences) as previously described (24). PBMCs were cultured in RPMI 1640 (Life Technologies) culture medium supplemented with $10 \%$ FCS, $2 \mathrm{mM}$ l-glutamine, $100 \mathrm{U} / \mathrm{ml}$ penicillin, and $100 \mathrm{~g} / \mathrm{ml}$ streptomycin-sulfate in 96-well round bottom plates (Thermo Scientific) at a density of $5 \times 10^{6}$ cells $/ \mathrm{ml}$. Freshly isolated PBMCs were starved in $1 \mathrm{ml}$ serum free RPMI 1640 culture media for $1 \mathrm{~h}$ at $37{ }^{\circ} \mathrm{C}$ in $5 \% \mathrm{CO} 2$ incubator. After starvation, cells were centrifuged at $500 \mathrm{xg}$ for $5 \mathrm{~min}$. Cell pellet was resuspended in appropriate amount of PBS pH7.4 with the final cell density of $1 \times 10^{7} / \mathrm{ml}$ per sample. Cells remain unstimulated or were stimulated with IFN- $\gamma\left(60 \mathrm{IU} / \mathrm{ml}\right.$ or $6 \times 10^{\wedge} 4 \mathrm{IU} / \mathrm{ml}$ in MilliQ, R\&D Systems, Abingdon, UK) or IFN- $\alpha\left(10^{4} \mathrm{IU} / \mathrm{ml}\right.$ in PBS, PeproTech, London, UK) for 5mins, $15 \mathrm{mins}$ and $30 \mathrm{mins}$. After stimulation, cells were fixed and reagent-permeabilized according to the manufacturer's protocol (PerFix EXPOSE (phosphor-epitopes Exposure)Kit; B26976, Beckman Coulter, Brea, CA).

\section{Flow cytometry}

For STAT1 phosphorylation analysis, subsequent to fixation and permeabilization, all samples were stained with Pacific Orange-conjugated mouse anti-human CD45(\#MHCD4530, clone HI30, Invitrogen, CA, USA), CD3 PerCP Cy5.5 mouse anti-human (\#332771, clone SK7, BD Biosciences, CA, USA), APC-conjugated mouse antihuman CD14(\#345787, clone M $\varphi \mathrm{P} 9$, BD Biosciences, CA, USA), PE-conjugated mouse anti-human-Stat1 (N-Terminus)(\#558537, BD Biosciences, CA, USA) and Alexa Fluor ${ }^{\circledR} 488$ conjugate rabbit antihuman Phospho-Stat1 (Tyr701) (\#9174S, clone 58D6, Cell Singling Technology, MA, USA) antibodies for 30mins in dark and measured with a BD FACSCanto ${ }^{\mathrm{TM}}$ II machine.

For CD64 membrane staining, freshly obtained PBMCs were starved in $1 \mathrm{ml}$ serum free RPMI 1640 culture media for $1 \mathrm{~h}$ at $37^{\circ} \mathrm{C}$ in $5 \% \mathrm{CO} 2$ incubator. After starvation, cells were centrifuged at $500 \mathrm{xg}$ for $5 \mathrm{~min}$, and $1 \times 10^{6}$ cells were seeded in 96 well round bottom plate with/without IFN- $\gamma$ (60IU/ml in MilliQ, R\&D Systems, Abingdon, UK) in RPMI 1640 culture medium containing $10 \% \mathrm{FCS}$ and antibiotics. Cells were cultured for $24 \mathrm{~h}$ at $37^{\circ} \mathrm{C}$ in $5 \% \mathrm{CO} 2$ incubator. All samples

were subsequently stained with Pacific Orange-conjugated mouse anti-human CD45(\#MHCD4530, clone HI30, Invitrogen, CA, USA), APC-conjugated mouse anti-human 
CD14(\#345787, clone M $\varphi$ P9, BD Biosciences, CA, USA), PE-conjugated mouse antihuman CD64(\#644385, clone 10.1, BD Biosciences, CA, USA) antibodies for 15mins in dark and measured with a BD FACSCanto ${ }^{\mathrm{TM}}$ II machine.

For CD119 membrane staining, PBMCs were thawed from liquid nitrogen one night before measurement at a cell density of $2 \times 10^{6} / \mathrm{ml}$ in RPMI 1640 culture medium supplemented with $20 \%$ FCS and antibiotics at $37{ }^{\circ} \mathrm{C}$ in $5 \% \mathrm{CO} 2$ incubator. All samples were subsequently stained with Pacific Orange-conjugated mouse anti-human CD45 (\#MHCD4530, clone HI30, Invitrogen, CA, USA), APC-conjugated mouse anti-human CD14 (\#345787, clone M $\varphi$ P9, BD Biosciences, CA, USA), PE-conjugated mouse anti-human CD119 (\#12-1199-42, clone GIR 208, eBioscience ${ }^{\mathrm{TM}}$ )) antibodies for $15 \mathrm{mins}$ in dark and measured with a BD FACSCanto ${ }^{\mathrm{TM}}$ II machine.

\section{SOCS1 expression}

Freshly isolated PBMCs were starved in $1 \mathrm{ml}$ serum free RPMI 1640 culture media for $1 \mathrm{~h}$ at $37^{\circ} \mathrm{C}$ in $5 \% \mathrm{CO} 2$ incubator. Around $2 \times 10^{5}$ to $3 \times 10^{5}$ cells were taken and remained unstimulated or stimulated for $0.5 \mathrm{~h}, 1 \mathrm{~h}, 2 \mathrm{~h}, 4 \mathrm{~h}$ and $6 \mathrm{~h}$ with IFN- $\gamma(60 \mathrm{IU} / \mathrm{ml}$ in MilliQ, R\&D Systems, Abingdon, UK) or IFN- $\alpha\left(10^{4} \mathrm{IU} / \mathrm{ml}\right.$ in PBS, PeproTech, London, UK) in a $37^{\circ} \mathrm{C}$ water bath. After stimulation, cells were harvested, and RNA was extracted using GenEluteTM Mammalian Total RNA Miniprep Kit (\#SLCC7097,Sigma-Aldrich) and reverse transcribed into cDNA using Reverse Transcriptase reaction with random primers (Thermo Fisher Scientific). Real-time quantitative PCR for SOCS1 and GAPDH was performed with SOCS1 primer/probe mix (Hs00864158_g1, Thermo Fisher Scientific) and GAPDH primer/probe mix (Hs99999905_m1, Thermo Fisher Scientific). All tests were run on a 7900HT Fast Real-Time PCR System(Thermo Fisher Scientific). GAPDH, the housekeeping gene expression, was used to normalize SOCS1 gene expression results.

\section{Data visualization and statistics}

Graph plots were made using Graphpad Prism V9.0. 


\section{Results}

Clinical description of 3 patients with MSMD

Patient 1

A 6-year old girl was admitted to our pediatric ward with progressive cough, fever and tachypnea unresponsive to repetitive courses of common antibiotics. She is the only child of healthy, nonconsanguineous Caucasian parents. She had a remarkable medical history with multiple hospital admissions $(>20)$ for bronchial hyperactivity, lower respiratory tract infections and a coarctectomy at the age of 2 .

A CT scan showed mediastinal and hilar lymphadenopathy and a large right upper lobe mass and lymph nodes compressing the trachea. A malignancy was suspected and in the work-up an abdominal ultrasound showed 3 focal lesions in the spleen and hepatomegaly. Because of concerns about her severely compromised airway, steroids were given, improving intraluminar tracheal diameter, after which a bronchoscopy with alveolar lavage and a lymph node biopsy was performed. Histological examination on the lymph node ruled out malignant lymphoma. On sputum as well as bronchoalveolar lavage material an auramine stain revealed acid-fast bacilli while the polymerase chain reaction (PCR) test remained negative for $\mathrm{M}$. tuberculosis complex. Patient 1 did not have any known contact with tuberculosis patients and was not immunized with BCG. Quantiferon as well as T-spot assay were inconclusive. Subsequent sputum and stomach fluid, lymph node biopsy and bone marrow culture revealed Mycobacterium avium. Subsequently, Patient 1 was treated with azithromycin, rifampicin, ethambutol. Amikacine was added for the first 2 months. She recovered after a year of treatment. Genetic testing for primary immunodeficiency (PID) was performed because of disseminated non-tuberculous mycobacterial infection. A diagnostic WES-based PID gene panel analysis revealed heterozygosity for a known pathogenic variant in the IFNGR1 gene NM_000416.2(IFNGR1):c.188T>G, p.(Val63Gly) (Figure 1A). However, no second variant was detected. RNA expression analysis on blood showed expression of the IFNGR1 transcript with the p.(Val63Gly) variant, but no transcripts from the second allele were detected (data not shown). Because the clinical phenotype was typical for MSMD, there was only expression from the allele with the p.(Val63Gly) variant, and functional analysis showed a defect in IFNGR signaling, we continued to search for a variant on the second allele. SNP array analysis showed decreased binding of one probe in exon 3 of IFNGR1, and a long range PCR using 
primers surrounding exon 3 showed an heterozygous insertion in this region. Sequence analysis showed that this concerned a complex insertion of the right arm of an AluY transposable element in exon 3: NM_000416.2(IFNGR1):c.210_211ins[136;A[?];207_211]). Parental segregation analysis showed that the two variants were bi-allelic. Plasma IFN- $\gamma$ level on admission was elevated: $311 \mathrm{pg} / \mathrm{ml}$.

\section{Patient 2}

A 7-year old boy was admitted to our pediatric ward with unexplained and recurrent low-grade fever, neck and leg pain, vasculitis-like skin lesions and polydipsia since several months. He is the first-born of non-consanguineous Caucasian parents and has one healthy brother. He had a remarkable medical history for recurrent bronchial hyperactivity, recurrent parotitis, parechovirusassociated radiculitis 3 years earlier and a period of severe cluster headache 9 months prior to admission.

A diagnostic work-up using brain MRI, CT scans of thorax and vertebrae, total body Xray studies and abdominal ultrasound showed a hypophysitis leading to diabetes insipidus, central hypothyreoidism and hypocortisolism, several bone lesions in skull, vertebrae and hip, as well hilar lymphadenopathy and a single lesion in the spleen. Tuberculin skin test showed induration of $18 \mathrm{~mm}$. Cultures of a bone lesion and sputum grew Mycobacterium persicum. Patient 2 started treatment with rifampicin, isoniazid and ethambutol as well as medication for his panhypopituarism which included hydrocortisone, levothyroxine and desmopressin and has improved very well. Genetic testing was performed because of disseminated non-tuberculous mycobacterial infection. A diagnostic WES-based PID gene panel analysis showed heterozygosity of two different variants in the IFNGR1 gene: a known pathogenic variant NM_000416.2(IFNGR1):c.373+1G>T, p.(?) and variant NM_000416.2(IFNGR1):c.110T >C, p.(Ile37Thr), classified as probable pathogenic (Figure 1A). Parental segregation analysis showed that the two variants were bi-allelic, supporting a presumed diagnosis of IFNGR1 deficiency. Serum IFN- $\gamma$ levels were undetectable.

\section{Patient 3}


A 1-year old Asian girl was seen at the outpatient clinic with a swollen right upper arm and axillary lymphadenopathy. She had had a BCG vaccination in that arm and the scar looked inflamed. Her previous medical history showed several episodes of bronchial hyperactivity and eczema. A CT scan showed multiple active hilar and axillary lymph nodes, a splenic lesion and osteolytic lesions of her right humerus, 2 ribs and a vertebral lesion in L2. Pathology of a bone biopsy from the right humerus showed lesions which could be compatible with Langerhans cell histiocytosis (LCH). Treatment for multifocal Langerhans cell histiocytosis was started using prednisone and vinblastine conform the International Collaborative Treatment Protocol for Children and Adolescents with Langerhans Cell Histiocytosis. The bone lesions showed improvement but new skin lesions appeared at the site of the BCG scar, in her hair and at her back The bone lesions showed some improvement but new skin lesions appeared at the site of the BCG scar, in her hair and at her back. Repeated CT scans now showed multiple bone lesions in scapulae, ribs and progression of hilar as well as axillary lymphadenopathy. Skin biopsies of the lesion at her back and head showed no signs of LCH but a granulomatous infiltration and grew Mycobacterium bovis BCG strain. The previous diagnosis of $\mathrm{LCH}$ was re-evaluated and a new diagnosis of disseminated BCG-itis was made. Treatment was started with rifampicin and isoniazid; with rapid improvement on both skin and bone lesions as well as on lymphadenopathy. Genetic testing for PID using a diagnostic WES-based PID gene panel was performed because of disseminated non-tuberculous mycobacterial infection and revealed a novel variant in the STAT1 gene: NM_007315.3(STAT1):c.1379A>T, p.(Asn460Ile) (Figure 1B). Parental investigation showed this was a de novo variant.

\section{IFNGRI expression is reduced in the patients with the IFNGR1 variants}

Since variants in IFNGRI can result in loss of expression of IFNGR1 we measured surface expression of IFNGR1 using the IFNGR1-specific monoclonal antibody (CD119, clone GIR-208). The expression of IFNGR1 was severely reduced on monocytes of Patient 1 (Figure 2), which is line with the study of Sologuren et al. who showed that patients carrying homozygous p.(Val63Gly) variant have reduced expression using the GIR-208 IFNGR1 antibody (25). The p.(Ile37Thr) variant identified in Patient 2 has only been described once (26), however it was not shown whether this variant results in reduced surface expression of IFNGR1. On the other allele, Patient 2 had the splice site variant c. $373+1 \mathrm{G}>\mathrm{T}$, which likely lead to prohibition of protein 
expression. Monocytes of Patient 2 had severely reduced expression of IFNGR1, suggesting that the p.(Ile37Thr) variant leads to reduced surface expression (Figure 2). The IFNGR1 surface expression was not reduced in Patient 3 (Figure 2).

Bi-allelic IFNGR1 variants leads to substantial reduction in STAT1 phosphorylation via IFN- $\gamma$ signaling in monocytes

The pathogenic variant p.(Val63Gly) in Patient 1, was described previously as leading to autosomal recessive partial IFNGR1 deficiency (26). Patients reported with this pathogenic variant had low levels of IFNGR1 expression on the cell surface and low binding of IFN- $\gamma$ to the receptor present on the cell surface. It was shown that stimulating monocytes with high doses of IFN- $\gamma$ resulted in a better IFN- $\gamma$ signaling, although still reduced compared to healthy controls (25). In line with this, we also observed that phosphorylation of STAT1 (pSTAT1) was absent in response to stimulation with low IFN- $\gamma$ concentration compared with healthy controls (Figure 3). This reduction in pSTAT1 could not be attributed to reduced STAT1 expression, since the levels of STAT1 in Patient 1 were in the normal range of the controls (Figure 4). Furthermore, the phosphorylation of STAT1 upon IFN- $\alpha$ stimulation was normal, confirming that the defect in STAT1 phosphorylation is specific for IFN- $\gamma$ signaling (Figure 5).

The splice variant c. $373+1 \mathrm{G}>\mathrm{T}$ in Patient 2 , has been described as pathogenic autosomal recessive variant and leads to complete IFNGR1 loss of function. The second variant p.(Ile37Thr) has also been described functionally as partial deficient $(12,13)$. Upon IFN- $\gamma$ stimulation, we found that the monocytes of Patient 2 had reduced expression of pSTAT1 compared with healthy controls (Figure 3). Also in Patient 2, STAT1 was normally expressed (Figure 4), and the phosphorylation of STAT1 upon IFN- $\alpha$ stimulation was normal (Figure 5), indicating a specific defect in IFN- $\gamma$ signaling in Patient 2.

The STAT1 gene variant p.(Asn460Ile) in Patient 3, is located in the DNA binding domain of STAT1 protein. We show that the protein expression of STAT1 is not affected (Figure 3), but the phosphorylation of STAT1 was slightly decreased. Moreover, it seemed to be delayed upon IFN$\gamma$ and IFN- $\alpha$ stimulation in monocytes compared to healthy controls (Figure 3 and Figure 5).

No upregulation of SOCS1or CD64 upon IFN- $\gamma$ stimulation

Page 11 of 21 
IFN-mediated STAT1 activation results in the transcription of IFN regulated genes, including SOCS1 and CD64. To further investigate the effect of the variants identified in the three patients, we determined if SOCS1 mRNA was upregulated upon stimulation with IFN- $\gamma$ or IFN- $\alpha$. While in healthy controls SOCS1 mRNA level clearly increased after stimulation, in Patient 1 and 2 no upregulation of SOCS1 was observed after stimulation with IFN- $\gamma$ (Figure 6 A). In contrast, IFNa stimulation induced high levels of SOCS1 mRNA expression in Patient 1 and Patient 2 (Figure 6B). These data confirm that there is a functional loss in IFNGR1 signaling in Patient 1 and 2, while signaling via the IFN- $\alpha$ receptor is still functional. In Patient 3, the expression of SOCS1 seems normal after $0.5 \mathrm{~h}$ of stimulation with IFN- $\gamma$, but was reduced after 1 and 2 hours of stimulation. We also measured CD64 membrane expression in monocytes after IFN- $\gamma$ stimulation in Patient 2. After 24h of IFN- $\gamma$ stimulation, there was no increase in CD64 surface expression, while in the healthy control CD64 expression increased after IFN- $\gamma$ stimulation (Figure 7). 


\section{Discussion}

We describe three patients with presentation of MSMD with new (combination of) bi-allelic or de novo variants in genes involved in the IFN- $\gamma$ /STAT1 signaling pathway. All three patients presenting with disseminated non-tuberculous mycobacterial (NTM) infections caused by $M$. avium, M. persicum and M. bovis BCG, respectively. On presentation none of these patients were previously known with a primary immune deficiency. A general approach in diagnosing patients with disseminated NTM infections has been proposed in literature using flow cytometry, lymphocyte immunophenotyping and sequencing of genes known to be involved in NTM host immunity $(16,26)$. Measurement of IFN- $\gamma$ plasma levels during infectious episodes can also be helpful as patients with complete IFNGR deficiency will display highly elevated plasma levels of IFN- $\gamma$ (27). However, since recent advances in technologies with regard to genomic analysis, a genotype-first approach for new patients with a suspicion of an inborn error of immunity has currently become a more typical approach (28). Since availability for rapid genetic testing is available in our clinical setting, we used whole exome sequencing with a PID gene panel as the first-line diagnostic approach.

In Patient 1, primary genetic analysis revealed only one heterozygous IFNGR1 variant. Because the clinical phenotype was typical for MSMD, RNA expression was only identified from the allele with the p.(Val63Gly) variant, and functional analysis showed a defect in IFNGR signaling, we continued to search for a variant on the second allele. This concerns the complex insertion of the right arm of an Alu-Y transposable element in exon 3 (c.210_211ins[136;A[?];207_211]) and could be identified by the combination of the SNP array and long range PCR. Insertions of Alu elements are rare and contribute to about $0.1 \%$ of human genetic disorders (29). They have previously also been described in patients with X-linked severe combined immunodeficiency and with X-linked agammaglobulinemia (30). In Patient 2, heterozygosity for two different, known pathogenic variants in the IFNGR1 gene were found. Bi-allelic different hypomorphic variants in this gene have not been described extensively in literature and the functional effects in an individual case are unpredictable. In the third patient, a de novo novel variant in STAT1 was found. AD STAT1 deficiency is a known cause of disseminated BCG infection and multifocal osteomyelitis, warranting molecular phenotyping in order to prove the pathological effect of this novel variant. These cases illustrate that interpretation of the significance of these novel 


\section{Zhou et al.}

(combination of) genetic variants in each of these cases can be a diagnostic challenge which we encounter more frequently using advanced genetics such as WES as first-line diagnostics. The genetic and functional analysis helps in determining the exact diagnosis, as the clinical symptomatology in Patient 2 and 3 could also fit with the diagnosis LCH. Furthermore, the analysis indicates also that we should continue the search for a second variant missed by WES-based analysis, when there is a strong clinical suspicion for the respective disorder.

In these cases, (functional) immunological phenotyping in combination with the outcomes of genetic testing is important in order to make a definitive diagnosis and predict the prognosis of an individual patient. This may lead to very specific therapeutic decisions. When the patient is considered to have the phenotype of complete autosomal recessive IFNGR1 deficiency for instance, allogeneic stem cell transplantation should be considered as a realistic therapeutic option, whereas a phenotype of partial IFNGR1 deficiency will be treated with watchful waiting and/or prophylactic antibiotics. Patients with novel combinations of variants such as in Patient 1 and 2 are diagnosed frequently, as novel variants in MSMD-related genes are still found (31-33). These cases should be carefully characterized using immunological phenotyping. Flow cytometric detection of the affected protein can be a rapid test in the clinical diagnosis of MSMD but is not always feasible or conclusive, depending on choice of antibodies $(25,34)$. For instance, a previous report indicate that patients homozygous for the p.(Val63Gly) variant have reduced surface expression of IFNGR when using the GIR-208 IFNGR1 antibody, but nearly normal using a different monoclonal antibody (MMHGR-1) (25). In Patient 1, the expression of IFNGR1 was significantly reduced on monocytes of Patient 1 using the IFNGR1 GIR-208 antibody, which means that the variant on the other allele, can either not be detected by the IFNGR1 GIR-208 antibody, or more likely is not expressed since we also could not detect mRNA transcripts from this allele. Furthermore normal protein expression does not necessarily preclude normal signaling function $(25,35,36)$.

In the patients presented in this study, who all had a clinical suspicion of MSMD and genetic variants in known MSMD genes, we chose to proceed with STAT1 phosphorylation and IFN- $\gamma$ induced SOCS1 and CD64 expression assays as markers for IFN- $\gamma$ signaling. The results of these assays helped in assessing the severity of the patients' phenotype. In Patient 1, the absence of STAT1 phosphorylation and SOCS1 mRNA expression combined with the elevated plasma IFN- 
$\gamma$ level and life-threatening presentation of disease led to consideration of allogeneic hematopoietic stem cell transplantation as a treatment option, for which patient and her parents are counselled. Patient 2 is still under treatment for his disseminated NTM infection and recovering very well. In Patient 3 the STAT1 phosphorylation was slightly decreased and is therefore alone not conclusive to confirm the pathogenicity of the p.(Asn460Ile) variant in STAT1. Therefore we also tested the upregulation of SOCS1 mRNA upon stimulation with IFN- $\gamma$. These results were more conclusive and showed a lack of upregulation of SOCS1. The asparagine at position 460 in STAT1 is located in the DNA binding domain, and crystal structures suggest that Asn 460 is the only amino acid that directly interacts via hydrogen bonds to DNA in the GAS sequence, which implies that this amino acid is crucial for STAT1 function $(37,38)$. Together, these functional data and the fact that the variant is de novo strongly suggest that Patient 3 has an AD STAT1 deficiency.

\section{Conclusion}

Patients presenting with disseminated NTM infection in early or late childhood with bi-allelic or non-conclusive genetic variants in genes involved in NTM immunity need to be phenotyped by functional assays of the IFNG/STAT1 signaling pathway in monocytes. These assays are helpful in assessing the extent of IFNGR pathway signaling defects and in clinical decision making in how to treat the patient. 


\section{Acknowledgements}

The research for this manuscript was performed within the framework of the Erasmus Postgraduate School Molecular Medicine.

\section{Declarations}

Funding: not applicable

Disclosure of conflicts of interest: The authors have no conflict of interest.

Availability of data and material: available on request

Code availability: not applicable

\section{Authorship contributions}

HIJ and CV designed study. ZZ, HIJ, ML, RB, TH, and WD performed experiments and analyzed the data. $\mathrm{IH}$ and $\mathrm{AB}$ performed the genetic analysis. $\mathrm{CV}, \mathrm{PF}, \mathrm{AR}, \mathrm{VD}, \mathrm{PH}, \mathrm{EZ}$ and $\mathrm{AB}$ collected clinical data. ZZ, HIJ and CV wrote the manuscript. All authors critically read the manuscript and provided feedback.

Ethical approval: Approval of the local medical ethical committee was obtained for a longitudinal study on patients with primary immunodeficiency.

Consent to participate: Informed consent was obtained from the parents of all patients.

Consent for publication: Informed consent for publication was obtained from all individual participants included in this manuscript. 


\section{References}

1. Okada S, Ishikawa N, Shirao K, Kawaguchi H, Tsumura M, Ohno Y, et al. The novel IFNGR1 mutation 774del4 produces a truncated form of interferon-gamma receptor 1 and has a dominant-negative effect on interferon-gamma signal transduction. J Med Genet. 2007;44(8):485-91.

2. Bustamante J, Boisson-Dupuis S, Abel L, Casanova JL. Mendelian susceptibility to mycobacterial disease: genetic, immunological, and clinical features of inborn errors of IFN-gamma immunity. Semin Immunol. 2014;26(6):454-70.

3. Jouanguy E, Altare F, Lamhamedi S, Revy P, Emile JF, Newport M, et al. Interferon-gammareceptor deficiency in an infant with fatal bacille Calmette-Guerin infection. N Engl J Med. 1996;335(26):1956-61.

4. Newport MJ, Huxley CM, Huston S, Hawrylowicz CM, Oostra BA, Williamson R, et al. A mutation in the interferon-gamma-receptor gene and susceptibility to mycobacterial infection. $\mathrm{N}$ Engl J Med. 1996;335(26):1941-9.

5. Casanova JL, Abel L. Genetic dissection of immunity to mycobacteria: the human model. Annu Rev Immunol. 2002;20:581-620.

6. Filipe-Santos O, Bustamante J, Chapgier A, Vogt G, de Beaucoudrey L, Feinberg J, et al. Inborn errors of IL-12/23- and IFN-gamma-mediated immunity: molecular, cellular, and clinical features. Semin Immunol. 2006;18(6):347-61.

7. Bustamante J, Arias AA, Vogt G, Picard C, Galicia LB, Prando C, et al. Germline CYBB mutations that selectively affect macrophages in kindreds with $\mathrm{X}$-linked predisposition to tuberculous mycobacterial disease. Nat Immunol. 2011;12(3):213-21.

8. de Beaucoudrey L, Samarina A, Bustamante J, Cobat A, Boisson-Dupuis S, Feinberg J, et al. Revisiting human IL-12Rbeta1 deficiency: a survey of 141 patients from 30 countries. Medicine (Baltimore). 2010;89(6):381-402.

9. Prando C, Samarina A, Bustamante J, Boisson-Dupuis S, Cobat A, Picard C, et al. Inherited IL-12p40 deficiency: genetic, immunologic, and clinical features of 49 patients from 30 kindreds. Medicine (Baltimore). 2013;92(2):109-22.

10. Hambleton S, Salem S, Bustamante J, Bigley V, Boisson-Dupuis S, Azevedo J, et al. IRF8 mutations and human dendritic-cell immunodeficiency. N Engl J Med. 2011;365(2):127-38.

11. Kong XF, Martinez-Barricarte R, Kennedy J, Mele F, Lazarov T, Deenick EK, et al. Disruption of an antimycobacterial circuit between dendritic and helper T cells in human SPPL2a deficiency. Nat Immunol. 2018;19(9):973-85.

12. Boisson-Dupuis S, Ramirez-Alejo N, Li Z, Patin E, Rao G, Kerner G, et al. Tuberculosis and impaired IL-23-dependent IFN-gamma immunity in humans homozygous for a common TYK2 missense variant. Sci Immunol. 2018;3(30).

13. Bogunovic D, Byun M, Durfee LA, Abhyankar A, Sanal O, Mansouri D, et al. Mycobacterial disease and impaired IFN-gamma immunity in humans with inherited ISG15 deficiency. Science. 2012;337(6102):1684-8.

14. Filipe-Santos O, Bustamante J, Haverkamp MH, Vinolo E, Ku CL, Puel A, et al. X-linked susceptibility to mycobacteria is caused by mutations in NEMO impairing CD40-dependent IL-12 production. J Exp Med. 2006;203(7):1745-59.

15. Haverkamp MH, van Dissel JT, Holland SM. Human host genetic factors in nontuberculous mycobacterial infection: lessons from single gene disorders affecting innate and adaptive immunity and lessons from molecular defects in interferon-gamma-dependent signaling. Microbes Infect. 2006;8(4):1157-66. 
16. Wu UI, Holland SM. Host susceptibility to non-tuberculous mycobacterial infections. Lancet Infect Dis. 2015;15(8):968-80.

17. Liau NPD, Laktyushin A, Lucet IS, Murphy JM, Yao S, Whitlock E, et al. The molecular basis of JAK/STAT inhibition by SOCS1. Nat Commun. 2018;9(1):1558.

18. McKusick-Nathans Institute of Genetic Medicine JHU, Baltimore, MD. OMIM. Online Mendelian Inheritance in Man, $\mathrm{OMIM}^{\circledR}$. Retrieved from https://omimorg/. 2021.

19. Jouanguy E, Lamhamedi-Cherradi S, Lammas D, Dorman SE, Fondaneche MC, Dupuis S, et al. A human IFNGR1 small deletion hotspot associated with dominant susceptibility to mycobacterial infection. Nat Genet. 1999;21(4):370-8.

20. Kerner G, Rosain J, Guerin A, Al-Khabaz A, Oleaga-Quintas C, Rapaport F, et al. Inherited human IFN-gamma deficiency underlies mycobacterial disease. J Clin Invest. 2020;130(6):3158-71.

21. Bousfiha A, Jeddane L, Picard C, Ailal F, Bobby Gaspar H, Al-Herz W, et al. The 2017 IUIS Phenotypic Classification for Primary Immunodeficiencies. J Clin Immunol. 2018;38(1):129-43.

22. Bousfiha A, Jeddane L, Picard C, Al-Herz W, Ailal F, Chatila T, et al. Human Inborn Errors of Immunity: 2019 Update of the IUIS Phenotypical Classification. J Clin Immunol. 2020;40(1):66-81.

23. Smit A HR, Green P. . RepeatMasker Open-3.0. RepeatMasker Open-30. 1996.

24. Brakke. Density Gradient Centrifugation: A New Separation Technique1. Journal of the American Chemical Society. 1951;73(4):1847-8.

25. Sologuren I, Boisson-Dupuis S, Pestano J, Vincent QB, Fernandez-Perez L, Chapgier A, et al. Partial recessive IFN-gammaR1 deficiency: genetic, immunological and clinical features of 14 patients from 11 kindreds. Hum Mol Genet. 2011;20(8):1509-23.

26. van de Vosse E, van Dissel JT. IFN-gammaR1 defects: Mutation update and description of the IFNGR1 variation database. Hum Mutat. 2017;38(10):1286-96.

27. Fieschi C, Dupuis S, Picard C, Smith Cl, Holland SM, Casanova JL. High levels of interferon gamma in the plasma of children with complete interferon gamma receptor deficiency. Pediatrics. 2001;107(4):E48.

28. French MA, Tangye SG. The Next Generation of Diagnostic Tests for Primary Immunodeficiency Disorders. The Journal of Infectious Diseases. 2019;221(8):1232-4.

29. Deininger PL, Batzer MA. Alu repeats and human disease. Mol Genet Metab. 1999;67(3):183-93.

30. Lester T, McMahon C, Van Regemorter N, Jones A, Genet S. 14.17 X-linked immunodeficiency caused by insertion of Alu repeat sequences. Journal of Medical Genetics. 1997;34(9S).

31. Bossi G, Errichiello E, Zuffardi O, Marone P, Monzillo V, Barbarini D, et al. Disseminated Mycobacterium Avium Infection in a Child with Complete Interferon-gamma Receptor 1 Deficiency due to Compound Heterozygosis of IFNGR1 for a Subpolymorphic Copy Number Variation and a Novel Splice-Site Variant. J Pediatr Genet. 2020;9(3):186-92.

32. Zhang $P$, Ying W, Wu B, Liu R, Wang H, Wang X, et al. Complete IFN-gammaR1 Deficiency in a Boy Due to UPD(6)mat with IFNGR1 Novel Splicing Variant. J Clin Immunol. 2021.

33. Ying W, Liu D, Dong $X$, Wang W, Hui X, Hou J, et al. Current Status of the Management of Mendelian Susceptibility to Mycobacterial Disease in Mainland China. J Clin Immunol. 2019;39(6):600-10.

34. Allende LM, Lopez-Goyanes A, Paz-Artal E, Corell A, Garcia-Perez MA, Varela P, et al. A point mutation in a domain of gamma interferon receptor 1 provokes severe immunodeficiency. Clin Diagn Lab Immunol. 2001;8(1):133-7.

35. Pierre-Audigier C, Jouanguy E, Lamhamedi S, Altare F, Rauzier J, Vincent V, et al. Fatal disseminated Mycobacterium smegmatis infection in a child with inherited interferon gamma receptor deficiency. Clin Infect Dis. 1997;24(5):982-4.

36. Jouanguy E, Dupuis S, Pallier A, Doffinger R, Fondaneche MC, Fieschi C, et al. In a novel form of IFN-gamma receptor 1 deficiency, cell surface receptors fail to bind IFN-gamma. J Clin Invest. 2000;105(10):1429-36. 
37. Chen X, Vinkemeier U, Zhao Y, Jeruzalmi D, Darnell JE, Jr., Kuriyan J. Crystal structure of a tyrosine phosphorylated STAT-1 dimer bound to DNA. Cell. 1998;93(5):827-39.

38. Basab Roy ZZ, Gary D. Stormo. Quantitative specificity of STAT1 and several variants. Nucleic Acids Research. 2017;45(14):8199-207. 


\section{Figures Legends}

Fig.1 Schematic representation of IFNGR1 and STAT1 proteins

Schematic overview of the exons in the IFNGRI gene (a) and STAT1 protein (b) indicating the different domains and genetic variants identified in the three patients. The variants of Patient 1 are indicated in green, from Patient 2 in purple and from Patient 3 in red. SP, signal peptide; TM, transmembrane domain. The binding location for JAK1 and STAT1 are indicated in yellow and blue respectively; ND, N-terminal domain; CCD, coiled-coil domain; DBD, DNA-binding domain; LD, linker domain; SH2, SH2 domain; TAD, transactivation domain.

Fig.2 Expression of IFNGR1 protein

Analysis of IFNGR1 (CD119, clone GIR-208) on patient CD45+CD3-CD14+ monocytes (blue) and healthy control (HC)(red), Patient 1 and Patient 2 showed reduced expression of IFNGR1 in monocytes compared to the $\mathrm{HC}$.

Fig.3 Phosphorylation of STAT1 in monocytes upon IFN- $\gamma$ stimulation Flowcytometric analysis of phosphorylated STAT1 (pSTAT1) protein in monocytes after stimulation with 60IU/ml IFN- $\gamma$. Patient 1 had no pSTAT1 expression, Patient 2 had severely reduced and Patient 3 had slightly reduced pSTAT1 expression. The histograms show a representative plots of 1 experiment (a). The median fluorescence intensity (MFI) for two independent experiments are shown in (b).

Fig.4 Expression of STAT1 protein

Expression of STAT1 protein in unstimulated monocytes measured by flow cytometry showed that the expression of STAT1 is not different compared to healthy controls. HC: $n=11$; Patient 1 : $\mathrm{n}=3$; Patient 2: $\mathrm{n}=4$; Patient $3: \mathrm{n}=4$.

Fig.5 Expression of phosphorylated STAT1 after IFN- $\alpha$ stimulation

Flow cytometric analysis of phosphorylated STAT1 (pSTAT1) protein in monocytes after stimulation with $10^{\wedge} 4 \mathrm{IU} / \mathrm{ml}$ IFN- $\alpha$. Patient 1 and 2 had normal levels of pSTAT1, while the expression of pSTAT1 was slightly reduced in Patient 3 after 5 minutes of stimulation. The 
histograms show a representative plots of 1 experiment (a). The median fluorescence intensity (MFI) for two independent experiments are shown in (b). Patient 1: $\mathrm{n}=1$; Patient 2: $\mathrm{n}=2$; Patient 3 : $\mathrm{n}=2$.

Fig.6 Expression of SOCS1 mRNA relative to GAPDH Expression of SOCS1 mRNA relative to GADPH in peripheral blood mononuclear cells after stimulation with IFN- $\gamma$ (HC: $\mathrm{n}=2$; Patients: $\mathrm{n}=2$ respectively) (a) or IFN- $\alpha$ (HC: $\mathrm{n}=1$; Patient 1 : $\mathrm{n}=1$; Patient 2: $\mathrm{n}=1$; Patient 3: $\mathrm{n}=1$ ) (b) showed that Patient 1 and 2 have no SOCS1 expression after IFN- $\gamma$ stimulation, while stimulation with IFN- $\alpha$ result in normal induction of SOCS1 expression. The expression of SOCS1 is reduced in Patient 3 upon IFN- $\gamma$ stimulation.

Fig.7 Membrane CD64 expression in Patient 2 after IFN- $\gamma$ stimulation Flow cytometric analysis of CD64 membrane expression on monocytes showed that Patient 2 does not upregulate CD64 after 24h stimulation with IFN- $\gamma$ in contrast to the healthy control (HC). HC: $\mathrm{n}=1$; Patient 2: $\mathrm{n}=1$. 


\section{Figures}

a

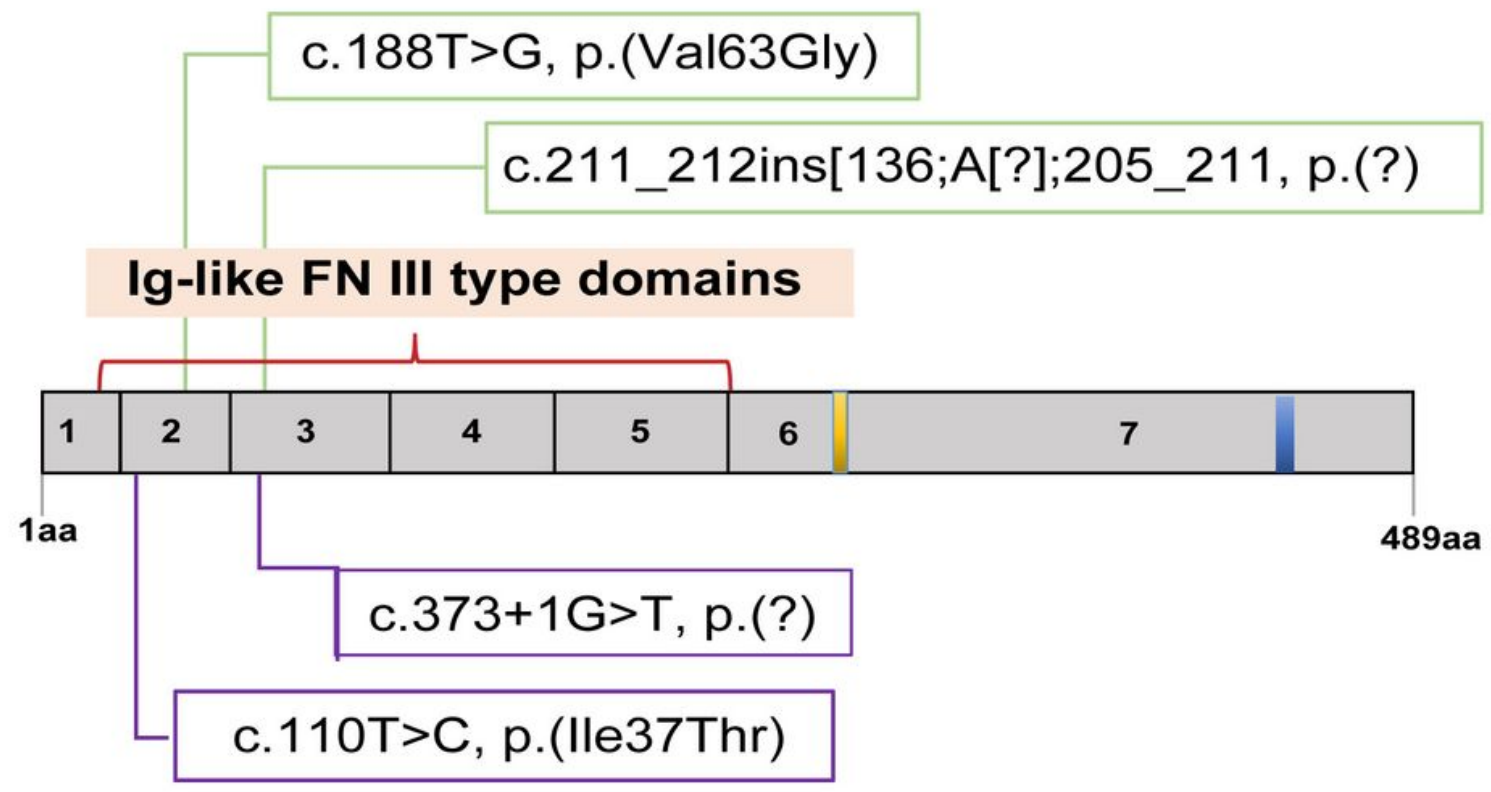

b

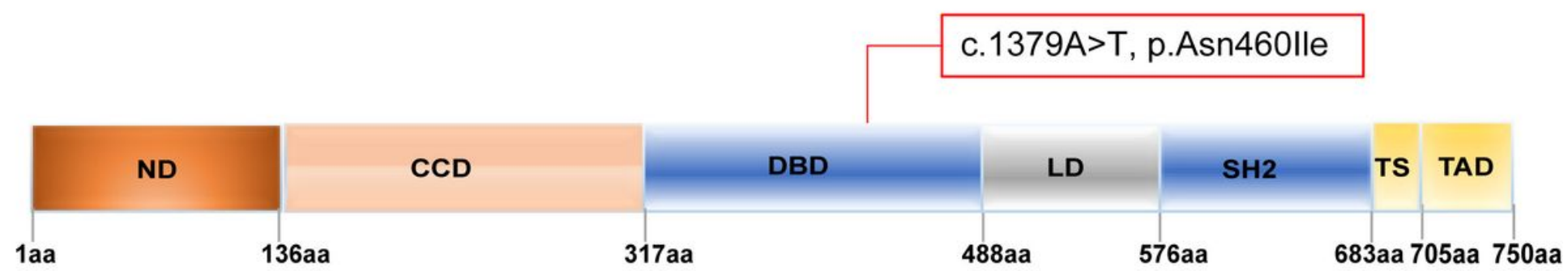

Figure 1

Schematic representation of IFNGR1 and STAT1 proteins Schematic overview of the exons in the IFNGR1 gene (a) and STAT1 protein (b) indicating the different domains and genetic variants identified in the three patients. The variants of Patient 1 are indicated in green, from Patient 2 in purple and from Patient 3 in red. SP, signal peptide; TM, transmembrane domain. The binding location for JAK1 and STAT1 are indicated in yellow and blue respectively; ND, N-terminal domain; CCD, coiled-coil domain; DBD, DNAbinding domain; LD, linker domain; $\mathrm{SH} 2, \mathrm{SH} 2$ domain; $T A D$, transactivation domain. 

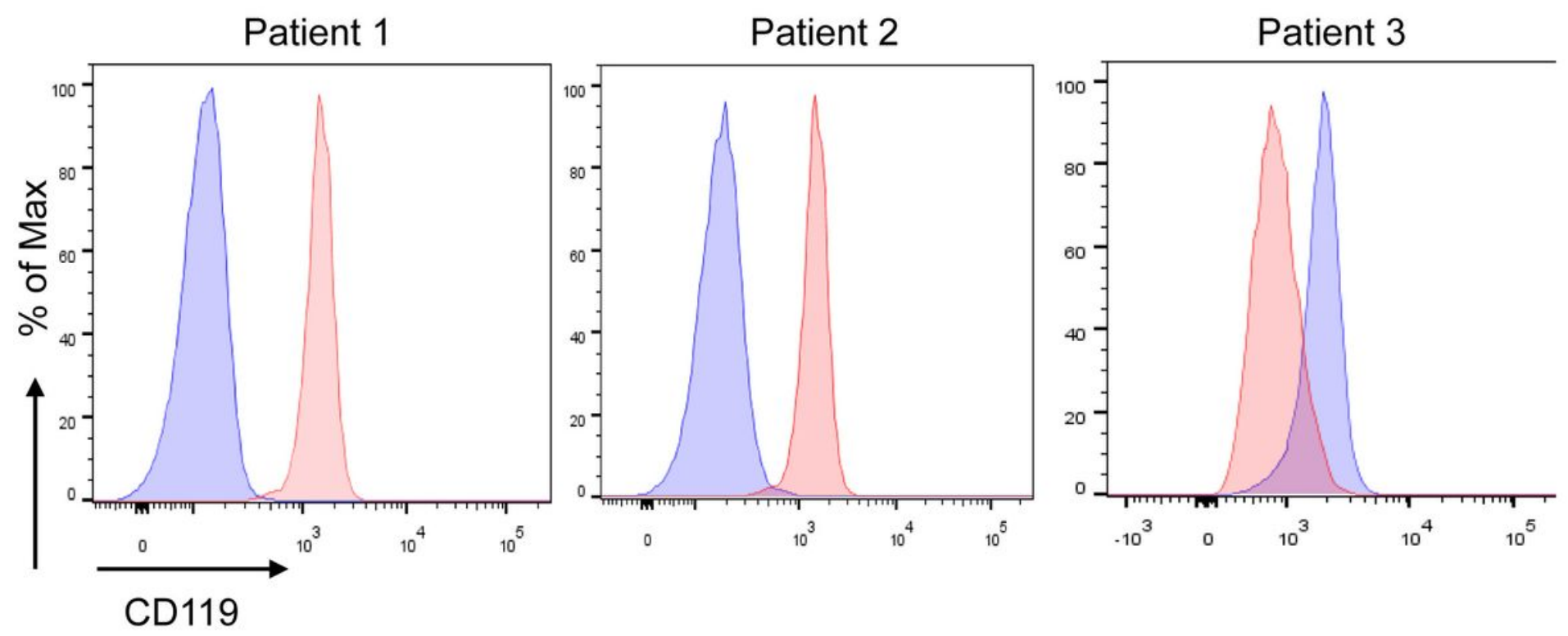

- HC CD14+ Monocytes
Patient CD14+ Monocytes

Figure 2

Expression of IFNGR1 protein Analysis of IFNGR1 (CD119, clone GIR-208) on patient CD45+CD3-CD14+ monocytes (blue) and healthy control (HC)(red), Patient 1 and Patient 2 showed reduced expression of IFNGR1 in monocytes compared to the HC. 
Patient 1

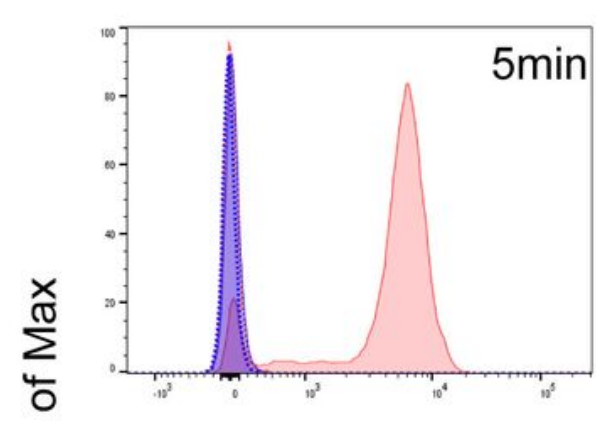

๙

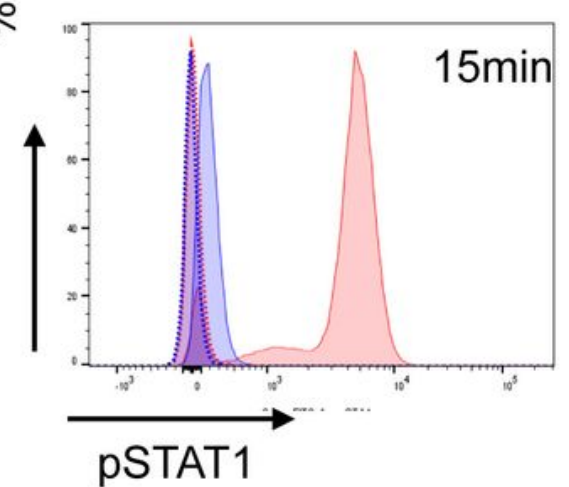

HC Unstimulated HC Stimulated
Patient 2
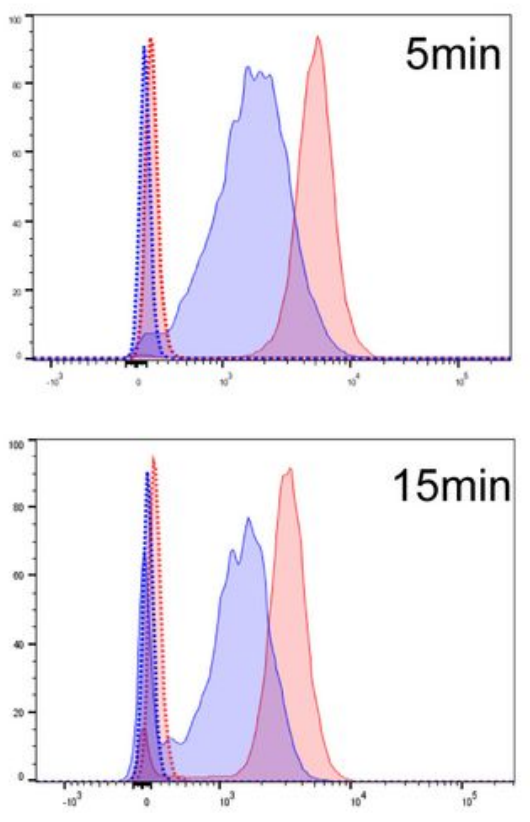

----. Patient Unstimulated
Patient 3
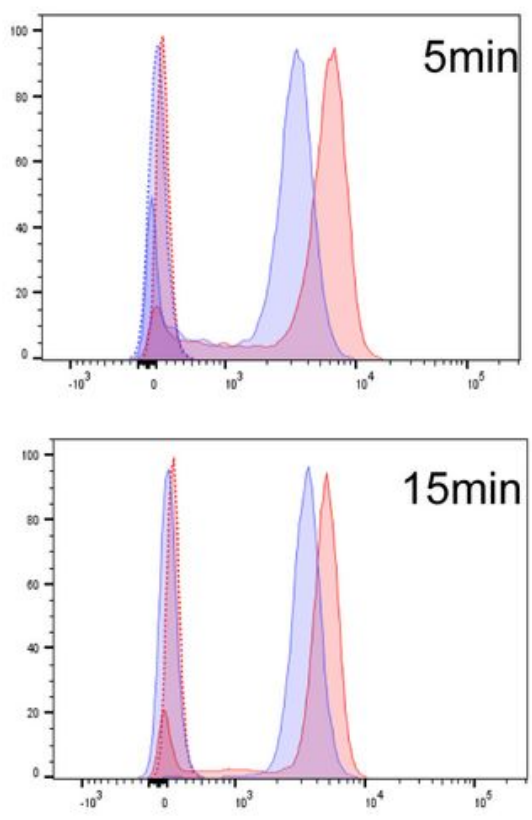

b

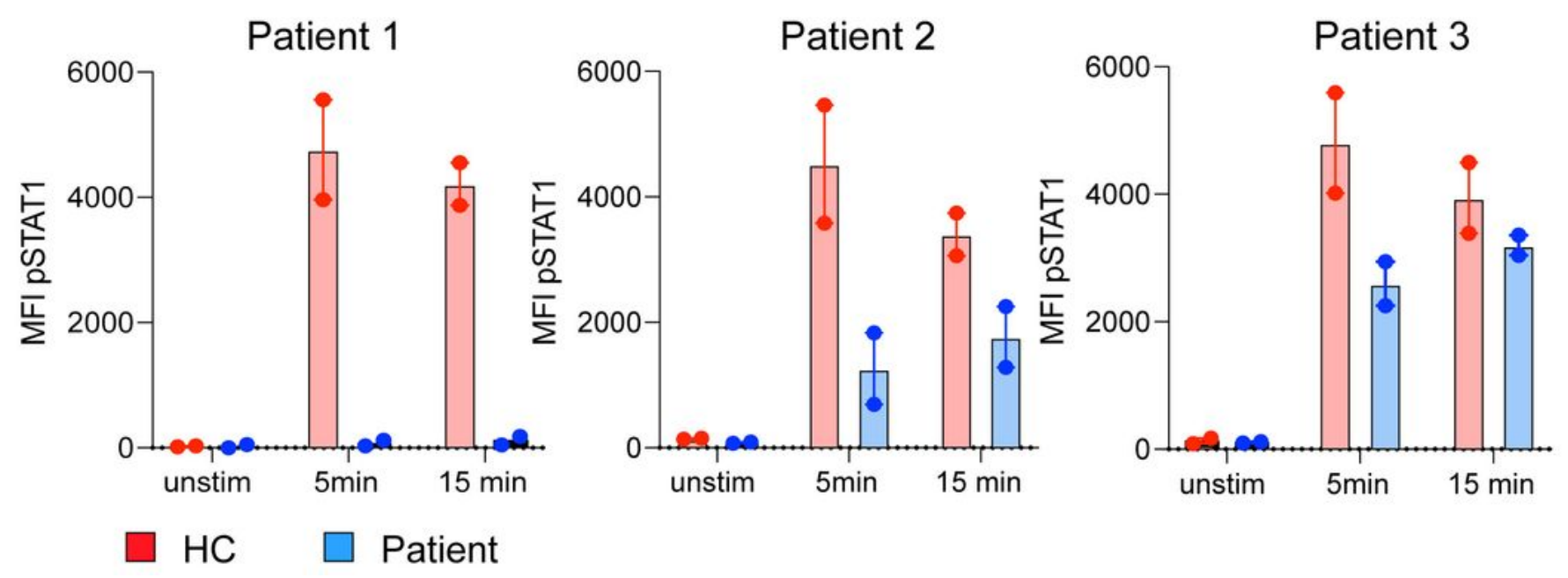

Figure 3

Phosphorylation of STAT1 in monocytes upon IFN- $y$ stimulation Flowcytometric analysis of phosphorylated STAT1 (pSTAT1) protein in monocytes after stimulation with $60 \mathrm{lU} / \mathrm{ml}$ IFN- $\gamma$. Patient 1 had no pSTAT1 expression, Patient 2 had severely reduced and Patient 3 had slightly reduced pSTAT1 expression. The histograms show a representative plots of 1 experiment (a). The median fluorescence intensity (MFI) for two independent experiments are shown in (b). 


\section{0}

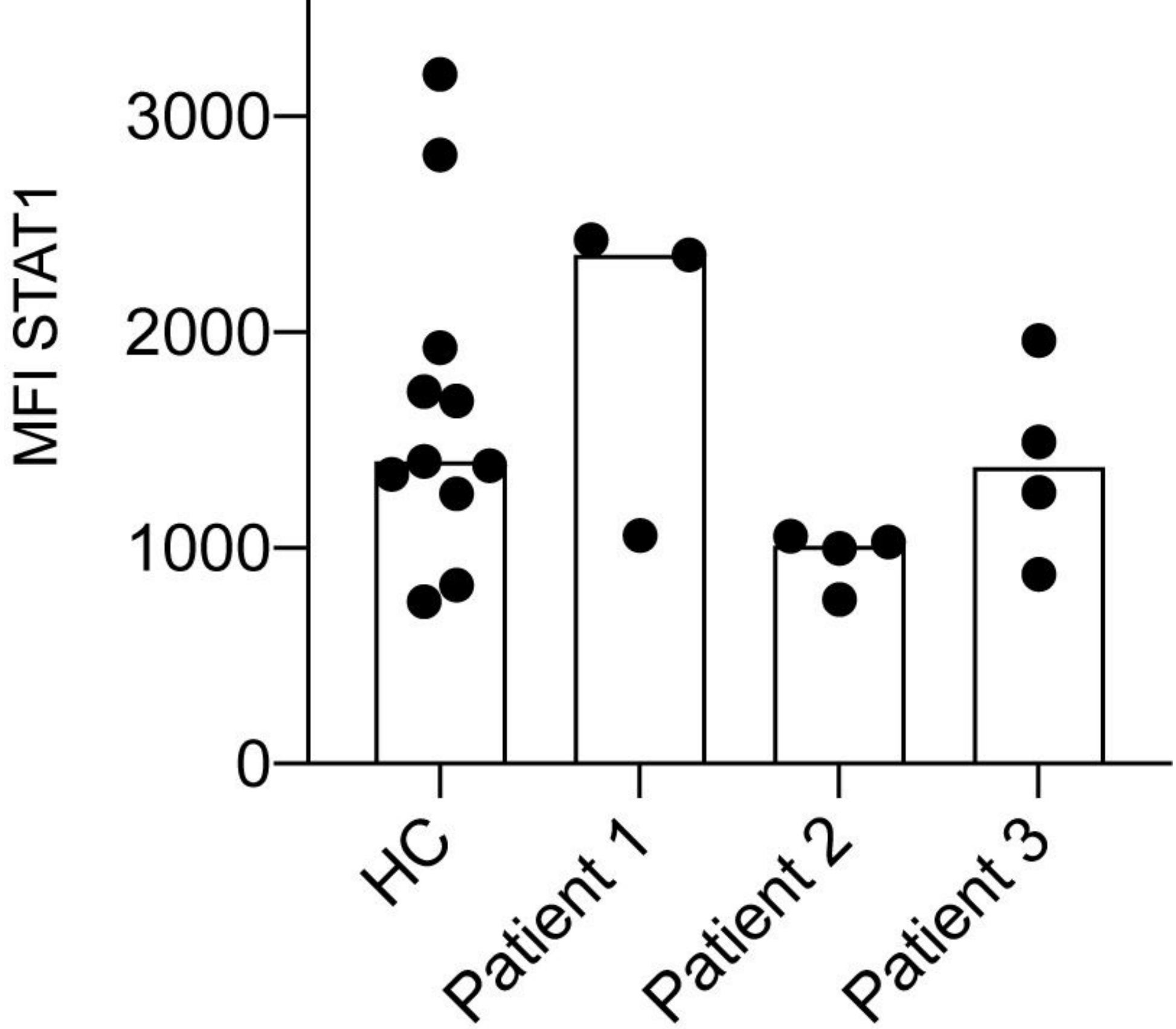

Figure 4

Expression of STAT1 protein Expression of STAT1 protein in unstimulated monocytes measured by flow cytometry showed that the expression of STAT1 is not different compared to healthy controls. HC: $n=11$; Patient 1: $n=3$; Patient 2: $n=4$; Patient 3: $n=4$. 

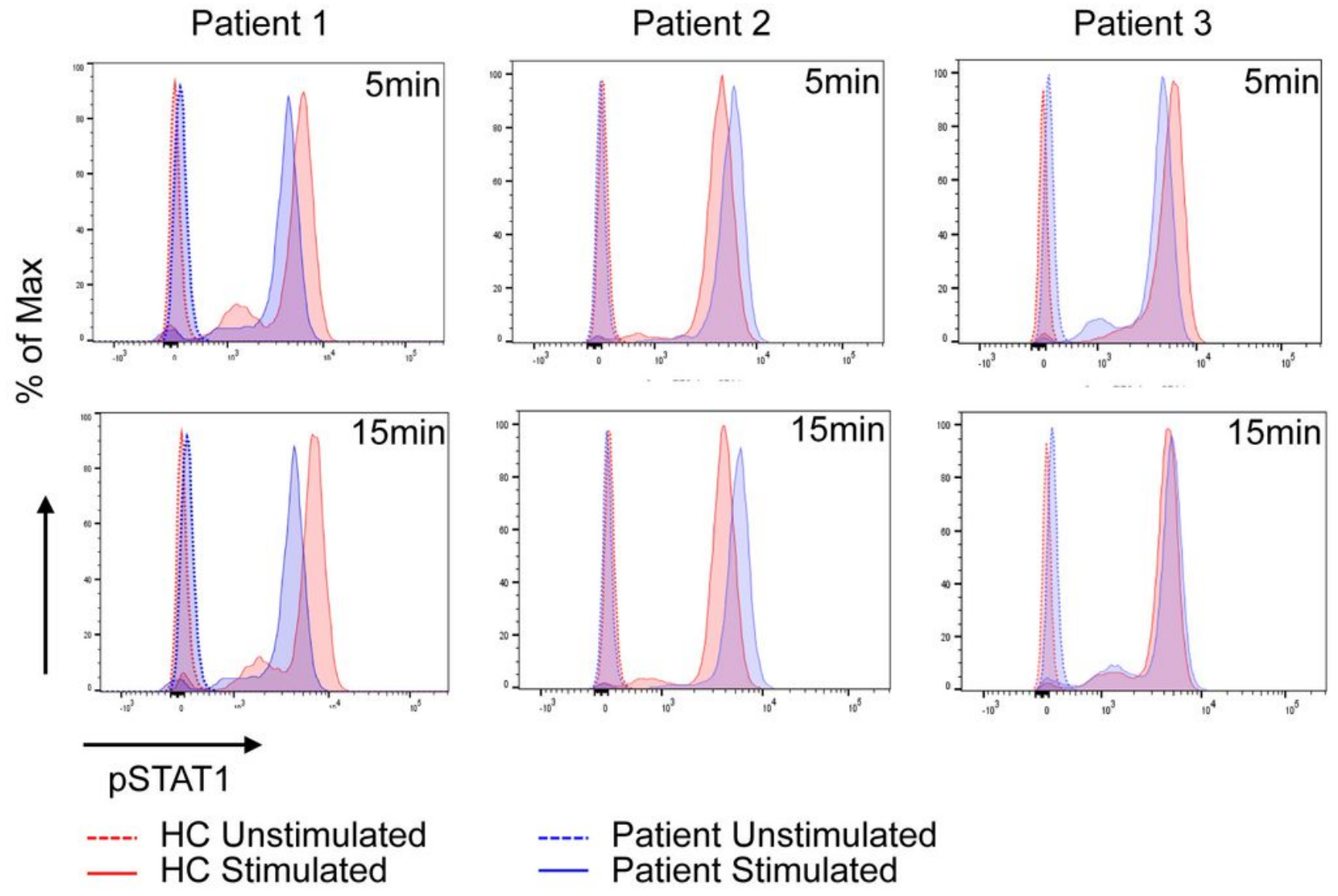

b

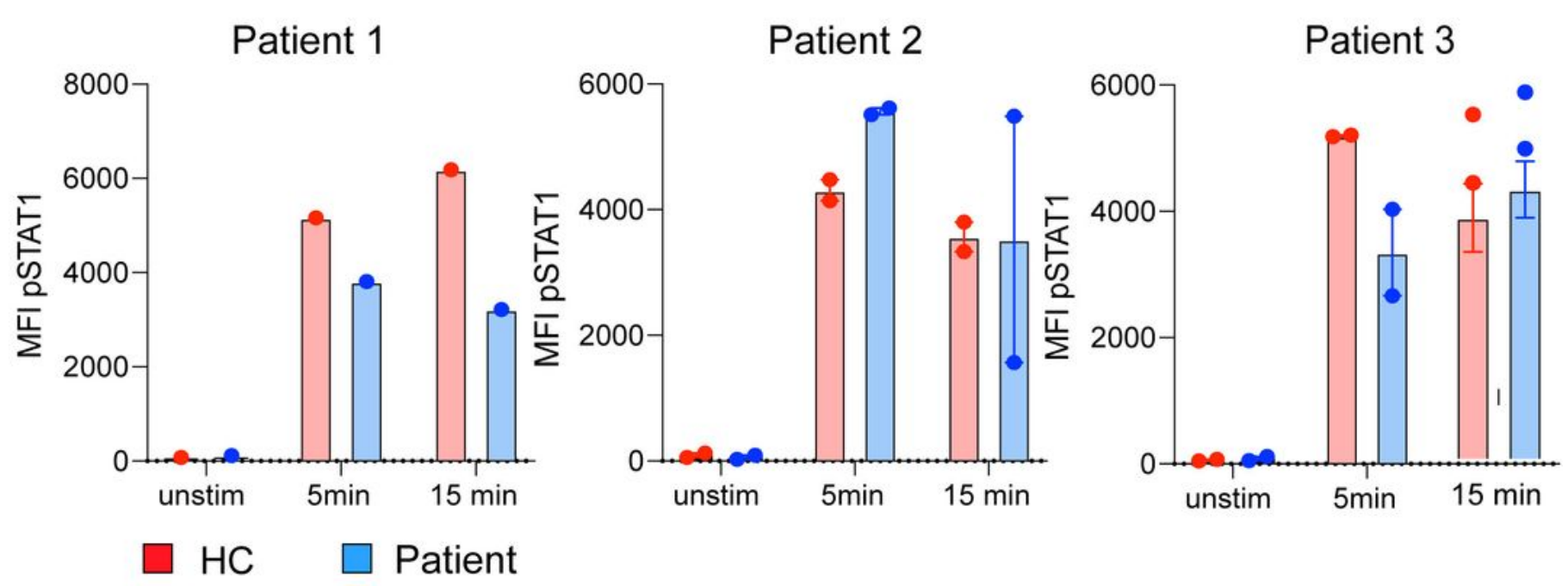

Figure 5

Expression of phosphorylated STAT1 after IFN-a stimulation Flow cytometric analysis of phosphorylated STAT1 (pSTAT1) protein in monocytes after stimulation with 10^4IU/ml IFN-a. Patient 1 and 2 had normal levels of PSTAT1, while the expression of PSTAT1 was slightly reduced in Patient 3 after 5 minutes of stimulation. The histograms show a representative plots of 1 experiment (a). The median 
fluorescence intensity (MFI) for two independent experiments are shown in (b). Patient 1: $n=1$; Patient 2: $\mathrm{n}=2$; Patient 3: $\mathrm{n}=2$.

a
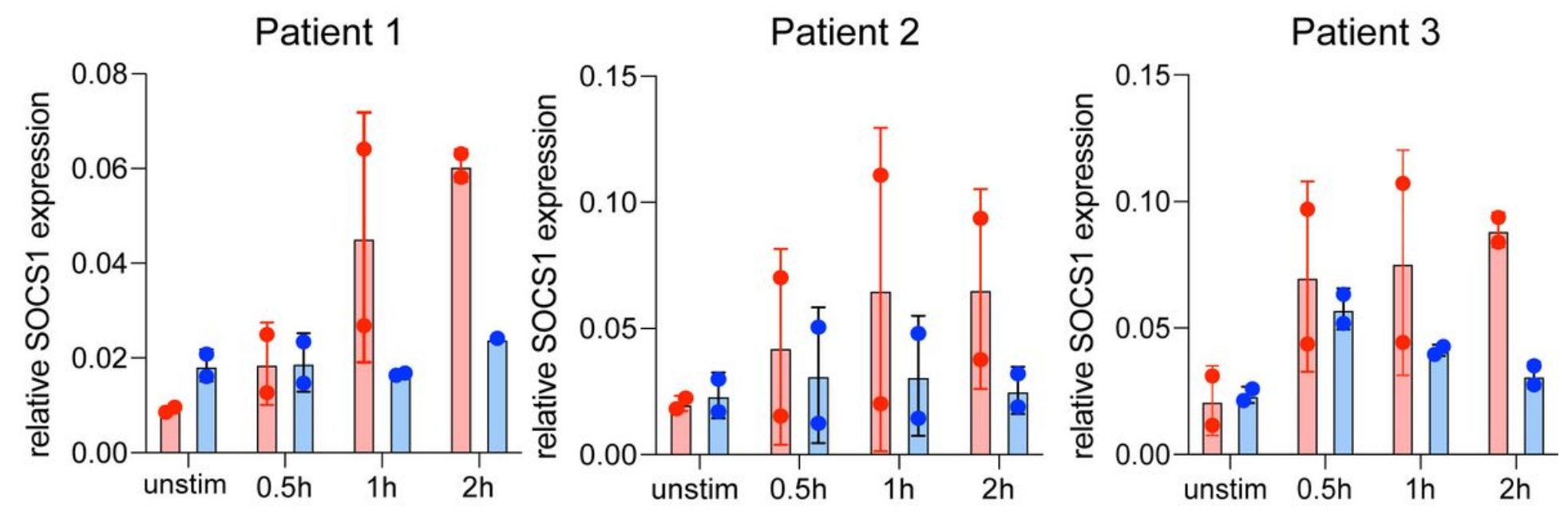

$\square \mathrm{HC} \quad \square$ Patient

b
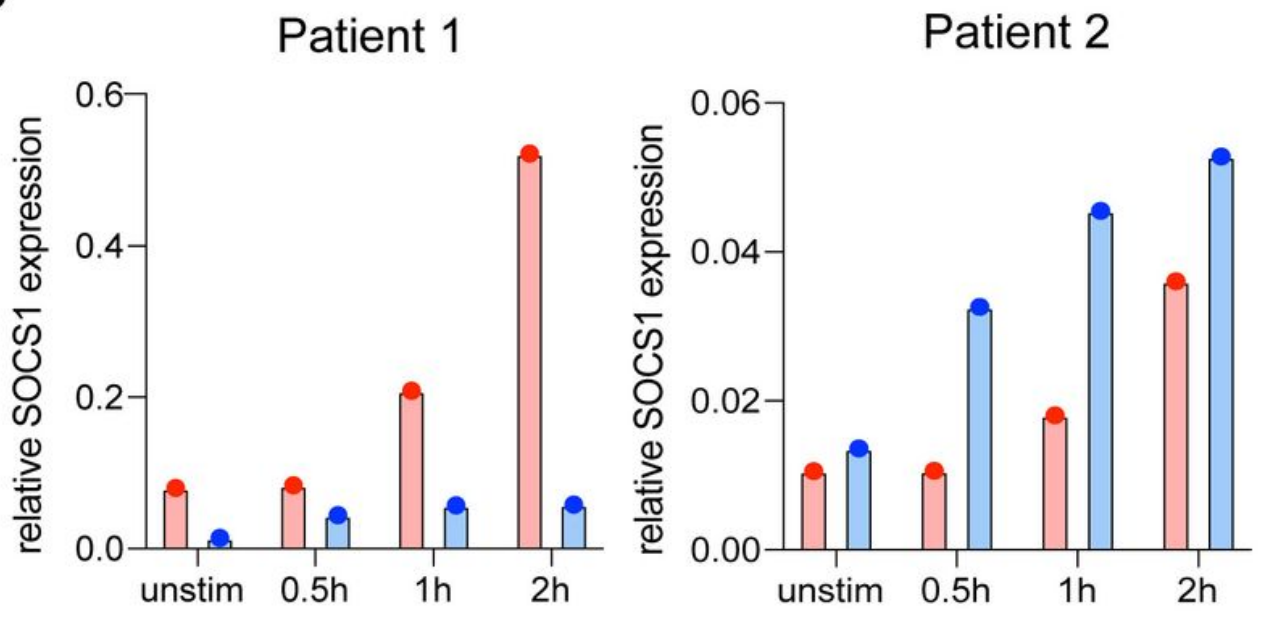

$\square$ HC $\square$ Patient

\section{Figure 6}

Expression of SOCS1 mRNA relative to GAPDH Expression of SOCS1 mRNA relative to GADPH in peripheral blood mononuclear cells after stimulation with IFN-y (HC: $n=2$; Patients: $n=2$ respectively) (a) or IFN- $a$ (HC: $n=1$; Patient 1: $n=1$; Patient 2: $n=1$; Patient 3: $n=1$ ) (b) showed that Patient 1 and 2 have no SOCS1 expression after IFN- $y$ stimulation, while stimulation with IFN-a result in normal induction of SOCS1 expression. The expression of SOCS1 is reduced in Patient 3 upon IFN-y stimulation. 
a

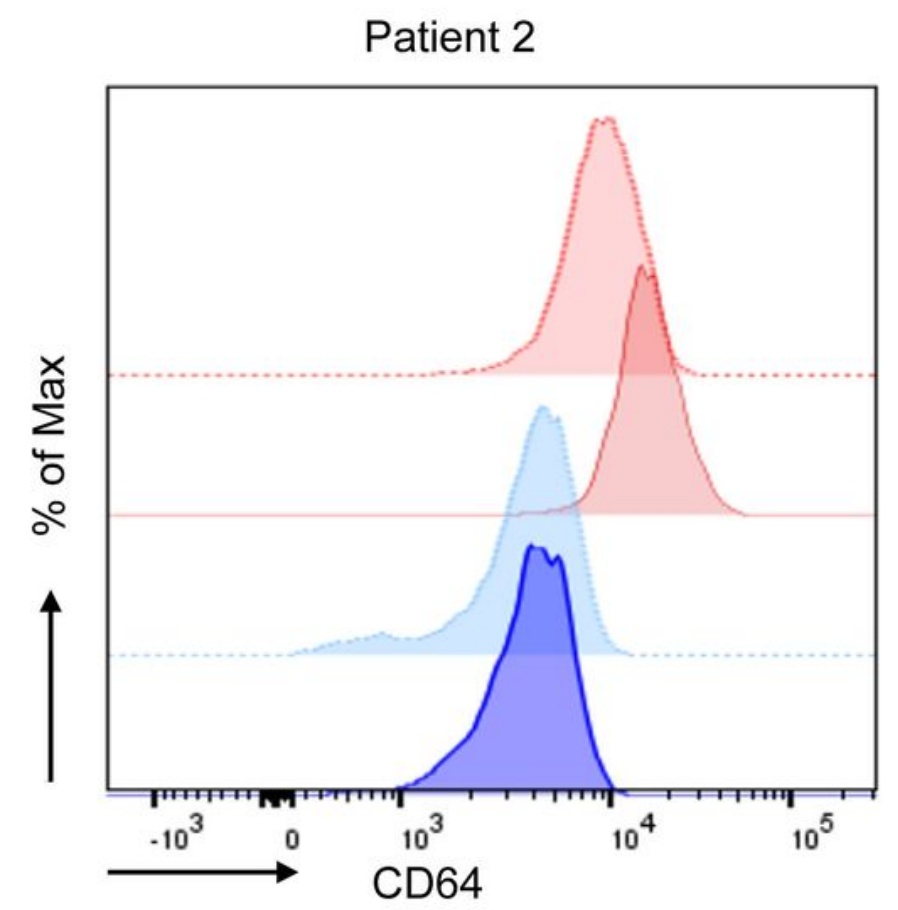

$\begin{array}{lll}\text {---- HC Unstimulated } & \text {----. Patient Unstimulated } \\ - \text { HC Stimulated } & - & \text { Patient Stimulated }\end{array}$

b

\section{Patient 2}

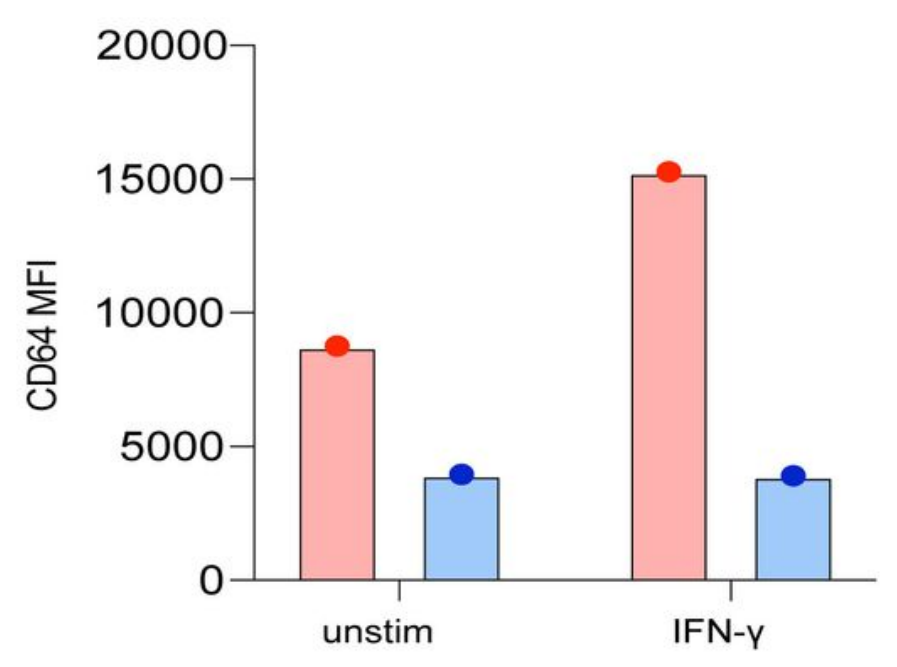

$\square \mathrm{HC} \quad \square$ Patient

\section{Figure 7}

Membrane CD64 expression in Patient 2 after IFN-y stimulation Flow cytometric analysis of CD64 membrane expression on monocytes showed that Patient 2 does not upregulate CD64 after 24h stimulation with IFN-y in contrast to the healthy control (HC). HC: $n=1$; Patient 2: $n=1$. 